\title{
Modeling Deployment of Enterprise Applications
}

\author{
Susanne Patig \\ University of Bern, IWI, Engehaldenstrasse 8, CH-3012 Bern \\ susanne.patig@iwi.unibe.ch
}

\begin{abstract}
Deployment comprises installing, activating and updating applications. The applications to be deployed usually require certain conditions that can refer to hardware capabilities, other software (dependencies), physical artifacts or configuration. Deployment planning aims at satisfying the applications' prerequisites without violating the hardware's capabilities. This paper presents the domain-specific language ADeL (Application Deployment Language) that was designed to describe and validate deployment plans. The ADeL metamodel was implemented within the Eclipse Modeling Framework (EMF) and contains a set of OCL constraints (implemented with the tool Topcased) to enable the automatic validation of deployment plans.
\end{abstract}

\section{Introduction}

As a result of mergers, acquisitions and evolving business needs, the application software (applications) and the IT infrastructure (hardware, system software and network) of a company change. Typical enterprise architecture (EA) approaches do not trace in detail the applications to the used IT infrastructure [2]. Such tracing information, however, is needed for IT consolidation, dependency analysis and the management of application portfolios [2].

This paper tries to close the gap between applications and IT infrastructure by dealing with deployment planning in data centers. Deployment comprises all activities that make some released software ready for use, namely installation, activation and updating [5]. Especially installation plays a key role as it has to obey software dependencies and sets up the initial relationship between applications and IT infrastructure. Installation presupposes the selection of the appropriate hardware, which does not only depend on the software's requirements, but also on deployment goals (e.g., performance or failure safety) and deployment restrictions (such as the current IT resource allocation or co-location constraints). Thus, deployment provides the basis for IT consolidation from a data center point of view.

Modeling deployment has several advantages: First, all information about the given hardware and its capabilities as well as about the applications and their requirements are described. Secondly, model validation can check the modeled deployment scenarios for realizability before installation starts and, thus, prevent installation breakdowns or even trigger the provisioning of missing hardware or software. Finally, software configuration [20], installation guidelines as well as virtualization layers [21] can be generated from the models. 
As a first step towards putting the advantages listed above into practice, this paper concentrates on modeling and validating deployment scenarios. In Section 2, the requirements of deployment planning are sketched; they are derived from real-world cases of deploying enterprise applications. Section 3 summarizes existing approaches for deployment modeling. As none of these approaches meets the requirements gathered in Section 2, Section 4 proposes a new domain-specific language called ADeL (Application Deployment Language) and applies it to a real-world case. Section 5 reflects on the language design process, and Section 6 contains the overall conclusions and an outlook.

\section{Requirements of Application Deployment}

The requirements of planning the deployment of complex applications in data centers are derived from two real-world cases, namely the installation of SAP SCM in the SAP UCC in Magdeburg and the installation of the content management system openCMS (http://www.opencms.org/) in the VLBA Lab Magdeburg ${ }^{1}$. During requirements elicitation, particular system's instances as well as documents related to installation were analyzed, and the staff involved in the installation process was interviewed. The complete description of the cases can be found in [17]; only the elicited requirements are listed in the following.

In detail, an approach that supports the deployment of complex applications in data centers must be capable to express (expressiveness requirements):

[Rq1] The available hardware and its technical characteristics (capabilities). The most important technical characteristics are CPU type (restricting the operating system) and CPU count as well as the sizes of random access memory (RAM) and hard disk (HD).

[Rq2] All that is to be deployed and has certain prerequisites, i.e., application components, system software or installation media. The prerequisites can refer to hardware capabilities, other software (i.e., dependencies), physical artifacts (e.g., executables, configuration files) or configuration activities (defining ports, IP addresses etc.). The objects to be deployed are called requirement units.

[Rq3] The direct or indirect assignment of requirement units to hardware; indirect assignments are realized transitively via intermediate requirement units.

[Rq4] Deployment constraints, e.g., whether or not some software units are allowed to run on the same server.

[Rq5] Choices in realizing some functionality (e.g., 'database functionality') by distinct software products (e.g., Oracle, DB2, MaxDB).

Interviews with the staff involved in the installation of complex applications made it clear that the expressive power reflected by the requirements [Rq1] to [Rq5] should be realized by a modeling language [Rq6] that is SImple, Extensible and General; I call this the SIEG principle. Simplicity [Rq7] means that only a small set of well separated

${ }^{1}$ All names of products are trademarks, service marks or registered trademarks of the respective companies. 
concepts should be used as the cognitive capacity of humans is limited [3]. Extensibility [Rq8] enables the adaptation of the new approach to specific deployment situations (by adding metamodel elements) and unanticipated usage scenarios (by adding (meta-) model transformations in model-driven development). As real-world application landscapes and hardware are heterogeneous, the new approach should be general [Rq9], i.e., independent of particular hardware, software and software architecture. Finally, the interviewed staff appreciated checking modeled deployment plans for their validity [Rq10] prior to installation as an important benefit of modeling.

The next section analyses whether or not the existing approaches in the field of deployment modeling satisfy the elicited requirements.

\section{Existing Approaches in the Field of Deployment}

Software deployment has been largely neglected in academic discussions. The earliest papers, e.g. [5], coin the key terms and classify existing technologies such as installers or package mergers. At that time, the aim was to automate deployment by tools, and modeling was just a prerequisite to achieve this aim. In contrast, the primary purpose of more recent approaches - such as ArchiMate [12] or UML Deployment Diagrams [15] - is to describe deployment scenario by models. These models are mainly used to document deployment or to discuss deployment scenarios with stakeholders, but not for automation.

Table 1 groups the existing approaches according to their (primary) aim. The term 'approach' is used as a generic name for tools, modeling languages and standards. Within each group, the approaches are ordered chronologically. The columns reflect the expressiveness requirements [Rq1] to [Rq4] of Section 2; requirement [Rq5] is omitted as it is not satisfied by any of the existing approaches. Concerning software, Table 1 distinguishes between conceptual units, such as components and packages and their relationships, and physical artifacts, e.g., executables or configuration files.

In the following, it is analyzed how the existing approaches meet the expressiveness requirements (see Section 2). Afterwards, the requirements related to the SIEG principle are investigated. Proprietary tools that automate the deployment of particular applications are disregarded here as they are not general [Rq9] and do not rely on modeling [Rq6].

The Software Dock framework [9] supports the deployment of software components via the Internet and assumes that software producer and consumer negotiate during deployment. The software to be deployed ('software families'2) is described by the Deployable Software Description (DSD), a language with textual concrete syntax that produces hierarchical schemas. The tool ORYA [13] allows the description and execution of a general deployment process (install, activate, deploy). Here, the focus is on automating the deployment workflow (involving legacy tools), but not on matching requirements of the software to the existing IT infrastructure. The tool $A D A G E$ (a research prototype) can deploy components on grids [11], given that the components follow the CORBA component model, a distinct programming model. Only ADAGE satisfies requirement [Rq1] as it provides constructs to describe hardware; see Table 1.

\footnotetext{
${ }^{2}$ The term is not used in the sense of software product lines.
} 
All automation-oriented approaches naturally have an IT view on deployment, i.e., they solely consider software, IT infrastructure and the corresponding assignments. In contrast, approaches that describe deployment can adopt an IT or a business view. The business view on deployment starts from business goals and business processes and links them to types of applications; it is typical for approaches stemming from the field of enterprise architecture (ArchiMate [12]) or go even beyond (MEMO ITML [8]). Both approaches provide distinct layers and diagrams; here, only the ones relevant to deployment are considered; see Table 1. In contrast, UML deployment diagrams [15], IBM topologies [14], [17] and the Common Information model ${ }^{3}$ (CIM) [6], which is implemented in configuration management databases (CMDB), represent the IT view on deployment. Some approaches support both views, see Table 1.

Table 1 illustrates that all approaches that aim at descriptions of deployment scenarios use distinct types of units to express the software to be deployed (e.g., UML [15]: specification, entity, process, service) and the IT infrastructure that is the target of deployment (e.g., UML [15]: execution environment, application server, client workstation, mobile/embedded device; ArchiMate [12]: device, system software; IBM topologies [14]: application server, WINDOWS/UNIX/LINUX/General OS etc.). Often these types are rather vaguely defined. Moreover, the description-oriented approaches share the usage of distinct link types (see Table 1), e.g., dependencies between software components, realization links that connect some conceptual deployment entity to another unit that will replace it (after deployment) and assignment links showing the IT infrastructure that will run (host) the deployed software unit. Often the semantics of the link types overlaps [14], [17]. In the resulting diagrams, the same units can be connected by several links, which affects understandability (see [17] for an example).

Altogether, all description-oriented approaches satisfy the requirements [Rq1] to [Rq3] as they can express hardware (IT infrastructure), software and the corresponding assignments. Minor restraints refer to the representation of artifacts: Explicitly representing artifacts is only possible with the UML by stereotypes such as 'script', 'source' and 'executable' [15]. In the IBM topologies, artifacts correspond to either attributes or additional constructs. Gaps exists for the other expressiveness requirements: The UML relies on the OCL [16] to specify any kind of deployment constraint [Rq4], whereas the IBM topologies support a limited set of constraint types by particular constructs [14]. Deployment choices [Rq5] are not covered by the existing approaches, except for indirect support within the IBM topologies (see [17]).

The postulated SIEG principle ([Rq7] to [Rq9]) is mostly not obeyed: Though the number of model elements in the approaches that automate deployment is small [Rq7], only the ORYA approach is general [Rq9]. Extending [Rq8] the automationoriented approaches for other deployment situations or usage scenarios is not possible.

In general, the large number of distinct unit and link types of the descriptionoriented approaches interferes with simplicity: In its common part, the CIM consists of 408 classes and 201 associations and is, thus, not simple [6]. However, because of being a standard, the CIM is general [Rq7] and adaptable to any deployment situation [Rq9]. The situation for the UML is analogous - the language is not simple, but both

\footnotetext{
${ }^{3}$ The CIM Specification V2.0 has been available sind June, 1999 [7].
} 


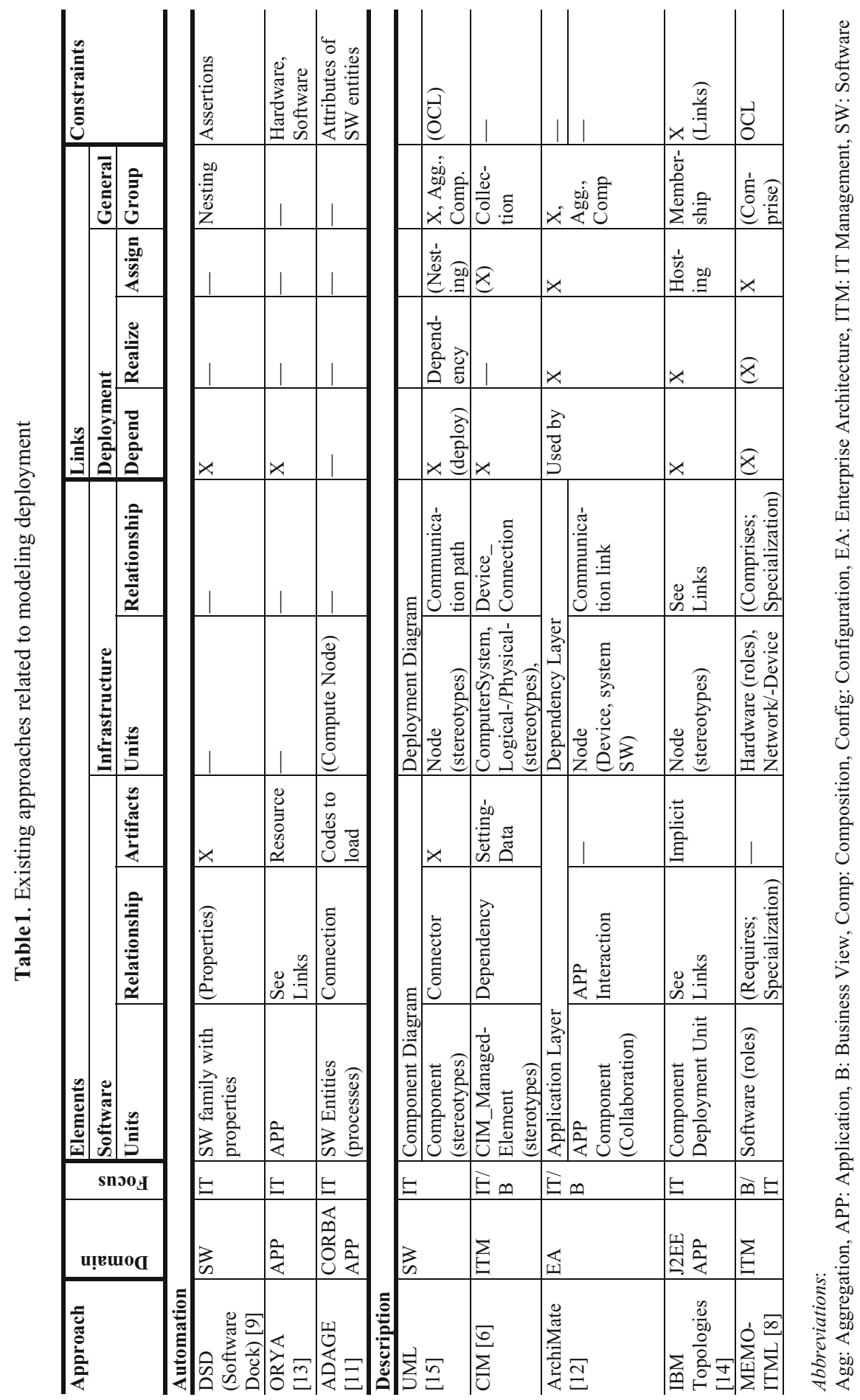


general and extensible (because of stereotyping and the available tools for modeldriven development). ArchiMate is not really simple and not really general (as it focuses on 'service-orientation' [12]), yet extensible. At the aggregated level, the number of constructs of the IBM topologies is comparatively small (units, components, deployment units, nodes, links), but they are broken down into a vast number of fine-grained, predefined constructs that mostly refer to IBM products. Thus, the IBM topologies are not general [14], [17], but they can be extended by userspecific constructs.

Validity checks [Rq10] are inherent in the automation-oriented approaches, but restricted to the particular tools and technologies these approaches support. Among the description-oriented approaches, only the IBM topologies offer some form of model validation by checking the modeled constraints [14], [17].

To sum it up, the main deficiencies of the existing approaches are missing simplicity [Rq7] as well as lack of support for deployment choices [Rq5], constraints [Rq4] and model validation [Rq10]. The domain-specific language ADeL proposed in the next section was designed to overcome these deficiencies.

\section{ADeL - The Application Deployment Language}

\subsection{ADeL Metamodel}

The ADeL metamodel consists of the abstract syntax depicted in Fig. 1 as well as a set of constraints that are expressed by OCL invariants.

The core ADeL metamodel elements are units; each unit can be linked (isLinked) to an arbitrary number of other units. As an abstract super class, a unit defines the common properties of both RUnits (requirement units, see [Rq2] in Section 2) and hardware: the name, an identifier id (if units cannot be recognized from their names), the type of CPU (CPU_type), the total number of CPU cores (CPU_count), the sizes of hard disk (HD) and random access memory (RAM). All properties except for the name are optional.

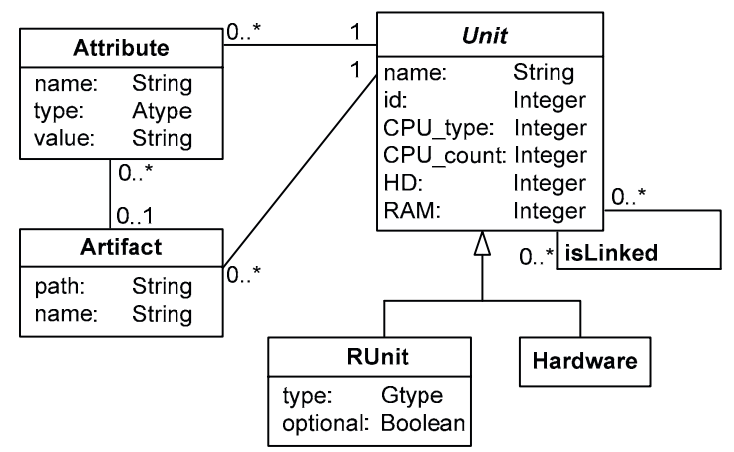

Fig. 1. Abstract syntax of the ADeL metamodel 
Units of the subtype hardware represent physical capabilities [R1] to host some RUnit(s). Basically, the prerequisites for RUnits can refer to hardware, software, physical artifacts or configuration (see [Rq2] Section 2). Hardware prerequisites are expressed by the properties listed above and paths to hardware [Rq3], whereas software prerequisites (dependencies) correspond to links (isLinked) between RUnits.

Units of the subtype hardware represent physical capabilities [R1] to host some RUnit(s). Basically, the prerequisites for RUnits can refer to hardware, software, physical artifacts or configuration (see [Rq2] Section 2). Hardware prerequisites are expressed by the properties listed above and paths to hardware [Rq3], whereas software prerequisites (dependencies) correspond to links (isLinked) between RUnits.

The predefined properties of units express standard deployment needs. Unforeseen prerequisites or capabilities can be modeled by attributes [Rq8]. A unit may be associated with an arbitrary number of attributes.

RUnits have the additional properties type and optional. The property type indicates whether a RUnit is elementary (GType $=E$ ), which is the default, or groups other RUnits. Groups of RUnits are either conjunctive (GType = A, i.e., all grouped RUnits must be deployed), disjunctive (GType $=$ o, i.e., at least one of the contained RUnits must be deployed) or exclusive (GType = X, i.e., one and only one RUnit of the group is to be deployed). Often such groups are conceptual, i.e., serve the purposes of structuring ADeL models or preparing deployment choices [Rq5]. The property optional describes whether or not some RUnit must be deployed at all.

Physical artifacts are needed for deployment execution, IT operations or result from configuration activities (e.g., configuration files, start profiles). They can be represented by the metamodel element artifact. A unit can be linked to any number of artifacts. The location of an artifact must always be given (property path), whereas the property name as well as associations to attributes are optional.

The ADeL metamodel is supplement by two OCL invariants that are independent of deployment, namely: (1) A node of the type RUnit must exist that is not the target of any association isLinked. This node is called the root node. (2) Identical hardware units agree in the values of their capabilities (CPU type and count as well as the sizes of RAM and HD).

The ADeL metamodel was implemented within the Eclipse Modeling Framework EMF 2.4.2 [4] and Eclipse 3.4 Ganymed. The current concrete ADeL syntax consists in to the graph provided by the EMF.Edit framework [4]; see Fig. 2 in Section 4.3.

\subsection{Deployment Constraints}

An instance of the ADeL metamodel, i.e., an ADeL model, corresponds to a deployment plan that successively assigns the RUnit of the root node (which is to be deployed) to hardware (leaf nodes); an example is given in Section 4.3. Only valid deployment plans can be effectuated. To be valid, a deployment plan (ADeL model) must satisfy 
all the RUnits' prerequisites (deployment constraints) without interfering with the hardware's capabilities (hardware constraints). Both groups of constraints are specified as OCL invariants [16] and explained in the following.

Deployment and hardware constraints rely on deployment paths, which exploit the association isLinked between units: A deployment path always starts at a RUnit and terminates at a unit of the types hardware or RUnit, respectively. In the first case, the start node is said to be deployed and undeployed otherwise.

Deployment constraints comprise the invariants [deployed] and [choice]. The invariant [deployed] requires that all RUnits that are not optional must be either linked to another unit (the child, which can be hardware) or belong to a nonelementary RUnit:

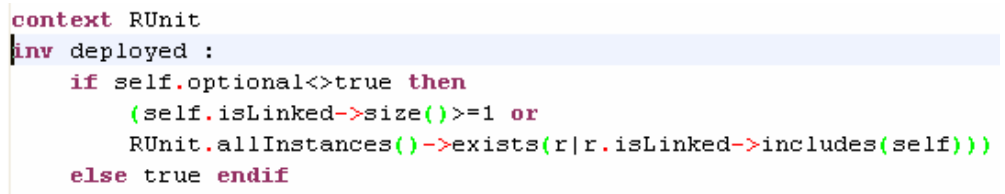

The deployment of non-optional, non-elementary RUnits is guarded by the invariant [choice]: If the group type (GType) of a non-elementary RUnit is $\mathrm{A} / \mathrm{O} / \mathrm{X}$, then for all/at least one/exactly one non-optional member(s) of the group a deployment path ending at a unit of the type hardware unit must exist:

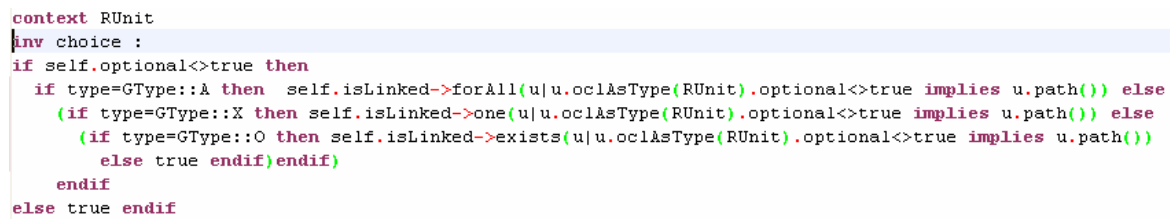

The recursive help function path() returns true when the last node of a deployment path is of the type hardware and false otherwise:

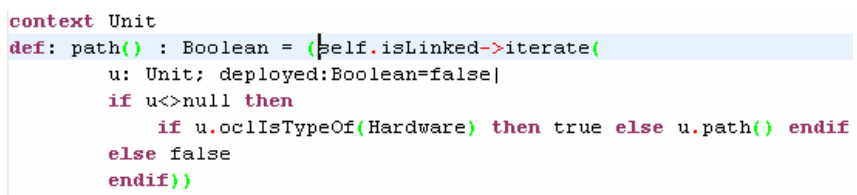

Hardware constraints, which are specified by the invariants [HD], [RAM], [CPU_count] and [CPU_type], guarantee that the aggregation of requirements along all deployment paths that target at the same hardware unit observe the hardware's capabilities. Consequently, these invariants must be specified in the context hardware, and navigation occurs along the reverse deployment path, i.e., from the leafs to the root of an ADeL model. Reverting the deployment path is achieved by iterating over all instances of the type RUnit and selecting parent RUnits that are linked with the corresponding (child) RUnit; see, e.g., the invariant [HD] :

context Hardware

inv HD: RUnit.allInstances ()$->\operatorname{select}(\mathrm{r} \mid \mathrm{r}$. isLinked->exists $(\mathrm{m} \mid \mathrm{m}$. name=self.name $))->$ collect $(u \mid u . \operatorname{aggrHD}(u . H D))->\operatorname{sum}()<=\operatorname{self} . \mathrm{HD}$ 
All invariants of hardware constraints use help functions for specific aggregations along the deployment path, i.e., (1) to sum up the required HD size (help function $\operatorname{aggrHD}($ ), see below), (2) to find the maximum required RAM size or CPU count or (3) to check the equality of the required CPU type. The invariants RAM, CPU_count, CPU_type and their help functions are specified analogously to the invariant HD.

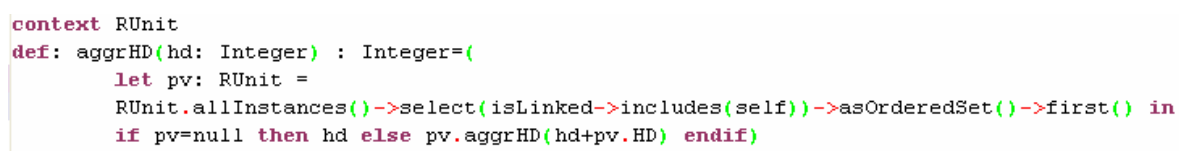

All OCL constraints for ADeL models are implemented with the Eclipse-based toolkit Topcased [22]. Topcased is integrated with the EMF modeling framework, includes a comfortable OCL editor and can evaluate both descriptive and statistic OCL constraints. The implemented ADeL OCL constraints are tied to the ADeL metamodel only and can be easily attached to all ADeL models. Moreover, standard OCL can be used to define additional constraints if necessary. Fig. 3 in the next section shows the result of evaluating the ADeL OCL constraints for the ADeL model of Fig. 2.

\subsection{Application Example}

Fig. 2 depicts the ADeL model for the deployment of SAP SCM (a real-world case investigated in [17]). On the right hand side of Fig. 2, examples for the properties of each metamodel element are given.

The SAP SCM deployment case has the following characteristics: The application software to be deployed (SAP SCM) consists of several RUnits (SAPKernel, DBSID, LID, OptID); an additional RUnit 'Install' expresses the installation requirements (installation media, JRE). The RUnit 'OptID' is optional. The 'SAPKernel' is a conjunctive RUnit (GType = A) since both the global instance 'SAPSID' and the central instance 'DVEBMG' as well as the 'C++ Runtime environment' must be installed. In contrast, the particular software products to be deployed for the RUnits 'DBSID', 'LID' and 'OptID' must be chosen from a set [Rq5]; thus, these RUnits are exclusive groups (GType $=\mathrm{X})$.

The graph structure of ADeL models supports the distribution of some RUnit over several hardware units, though this did not happen in the real-world case. The particular deployment of a RUnit is visible from the deployment path to a hardware unit. For example, the RUnit 'DBSID' is realized by the RUnit 'Oracle 10.2' (operating system 'HP-UX 11.23') and installed on the hardware unit 'HP Integrity rx8620'. The same hardware unit also hosts the RUnit 'LID' that requires the RUnit 'HP-UX 11.23' as operating system. In general, a hardware unit can host more than one RUnit.

The SAP SCM deployment case in Fig. 2 uses attributes and artifacts. For example, the attribute 'SWAP', which is associated with the RUnit 'SAPKernel', expresses the additional requirement of 20 Gigabyte (GB) of SWAP space. (All sizes are specified in GB in this paper). Moreover, the RUnit 'SAPSID' is associated with artifacts that specify the locations (path) of the directory / sapmnt and of the start profiles; the names of the artifacts help in distinguishing them from each other. 


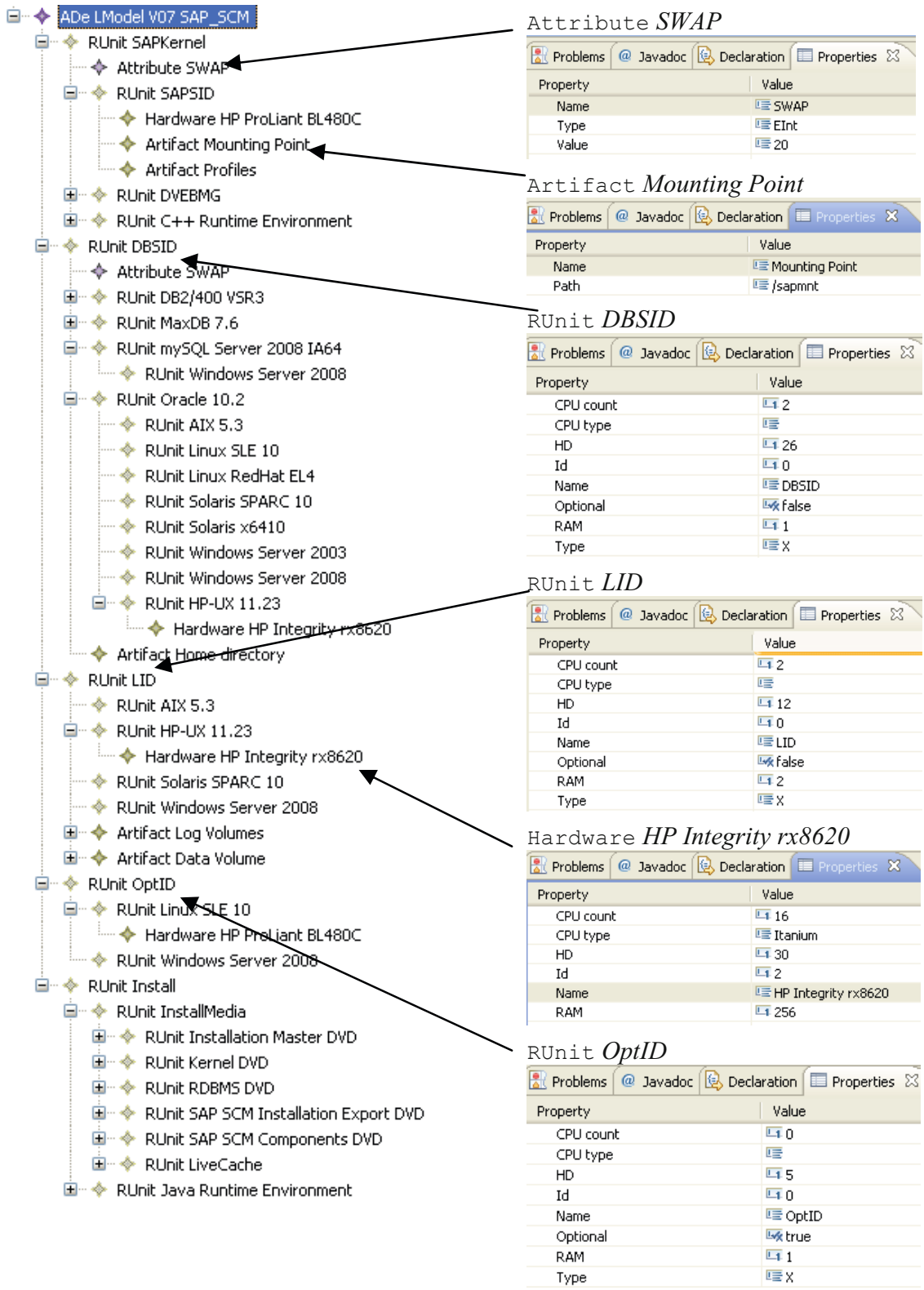

Fig. 2. Concrete syntax of the ADeL metamodel for the example of SAP SCM (extract)

Note that it is not necessary to detail all properties of units in an ADeL model as the ADeL OCL statements filter out null values. Thus, ADeL models can concentrate on the information essential for a deployment scenario.

The results of evaluating the ADeL OCL constraints for the ADeL model of Fig. 3 with the tool Topcased are shown in Fig. 3: For the purpose of illustration we assume 
that the hardware unit 'HP Integrity rx8620' already hosts other applications so that only 30 GB HD are available. The hardware unit 'HP Integrity rx8620' is the target of two deployment paths (from the RUnits 'DBSID' and 'LID', respectively; see Fig. 2). Along each path, the required hard disk space $\mathrm{HD}$ is smaller than the available $\mathrm{HD}$ of the hardware unit 'HP Integrity rx8620', namely 26 GB and 12 GB for the paths starting from the RUnits 'DBSID' and 'LID', respectively. However, as both RUnits are to be installed on the same hardware, the aggregated HD requirement amounts to $38 \mathrm{~GB}$, which exceeds the HD capacity of the hardware 'HP Integrity rx8620'. For that reason the evaluation of the invariant [HD] fails in Fig. 3. All other ADeL OCL constraints are observed by the deployment plan of Fig. 2.

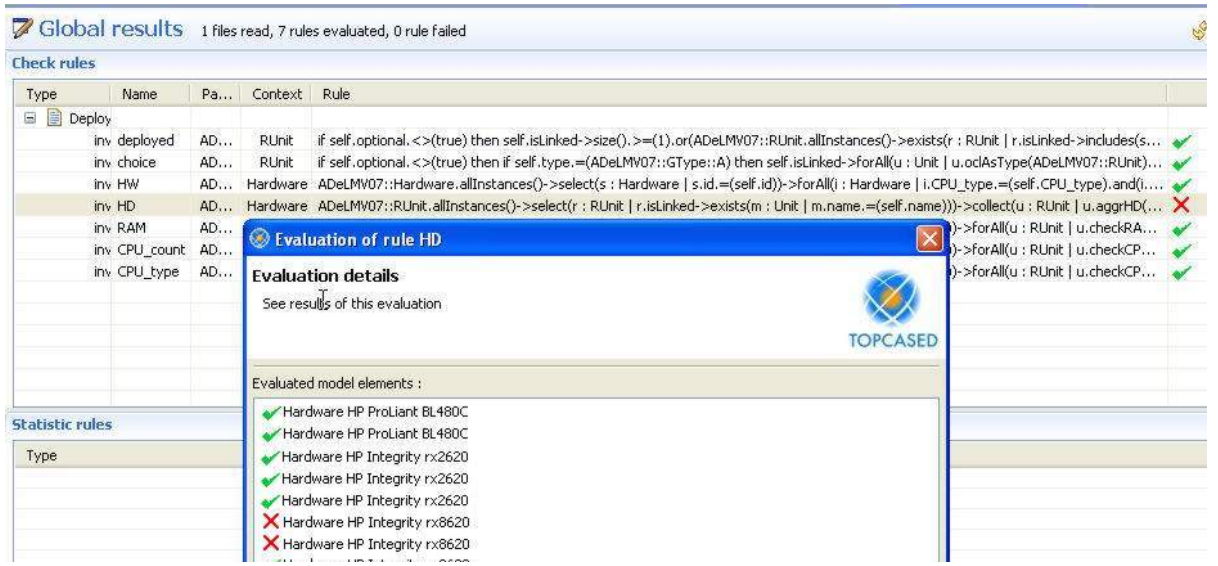

Fig. 3. Evaluation of the ADeL OCL constraints for the sample ADeL model

\section{Reflections on the ADeL Design Process}

The current version of the ADeL is the result of several major revisions. In this section I reflect on them as they possibly hint at generic issues in the design of domain-specific languages.

The advantages of deployment modeling listed in Section 1 call for methods and tools from model-driven software development. For that reason, the first version of the ADeL was a UML profile. However, the depth of inheritance within the UML infrastructure [23] turned out to be burdensome. To get rid of all the unnecessary, inherited semantics, a distinct, 'pure' ADeL metamodel was built, which can be seen as a UML profile at the level of Infrastructure: Core: :Abstractions: : Classifier [23].

Probably inspired by the existing deployment modeling approaches (see Table 1 in Section 3), the first versions of the ADeL metamodel were rich in specific types of units and links. For example, the metamodel element unit was specialized in abstract and concrete unit. Concrete unit contained the subtypes hardware and software, whereas abstract unit consisted of the subtypes concept (elementary unit) and set - to structure deployment plans. Moreover, it 
was distinguished between the link types membership, requires and realizes. Though facilitating modeling by the specific semantics, the extensive type vocabulary became heavy in writing OCL constraints: Basically, casting was possible between types of units, but made the OCL statements rather complicated. Even more severe were the problems in navigating along deployment paths that rely on distinct link types. As a result, the final version of the ADeL does not differentiate between link types and has only one layer of inheritance. Note that no information is lost by using only the generic association isLinked-as the units that are connected define the semantics.

Though the OCL is a natural choice to express constraints [Rq4] in the field of model-driven development, it is probably not the best one for reverse navigation in graphs: The OCL statements defining these navigations require the usage of the predefined operation allinstances(), which is deprecated because it makes navigation difficult to understand [19] and increases the worst case complexity of OCL evaluation [1]. However, the latter argument can be mitigated by the fact that the number of instances of each type within ADeL models is small, even in real-world deployment scenarios.

Altogether, the recursive functions to navigate in the graphs of the ADeL models are procedural rather than declarative (see, e.g., the help function path ( ) in Section 4.2). Eventually this observation indicates that the OCL is not the appropriate means to validate deployment plans.

\section{Criticism and Future Research}

Probably the main objection to the ADeL approach is over simplification, as the abstract syntax is a graph of linked units. However, recent research on enterprise architecture has shown that linked units are the basis to generate any kind of EA visualization and to exchange EA models between tools [10].

By using the ADeL language, it is very easy to assign the evolving set of applications in a data center to the existing IT infrastructure and, at the same time, to assure the validity of these assignments. In detail, the domain-specific language ADeL satisfies all the requirements listed in Section 2: The assignment [Rq3] of software or other units [Rq2] to some hardware [Rq1] as well as deployment choices [Rq5] can be described and validated [Rq10]. To specify other deployment-related constraints [Rq4], the OCL [22] can be used. Because of relying on just three mandatory, non-abstract metamodel elements (RUnit, hardware, isLinked), the modeling language ADeL [Rq6] is simple [Req7], yet extensible [Req8]- by the metamodel elements attribute and artifact. Moreover, all metamodel elements are independent of any real-world entities, tools and modeling languages and, thus, generic [Req9]. Finally, because of staying within the frameworks of model-driven software development, the ADeL language can be extended to a full-fledged approach of model-driven deployment that generates, e.g., installation guidelines and virtualization layers. Such an extension is a goal for future research.

The application of the ADeL to model and validate real-world deployment plans was shown. Currently, a more sophisticated editor is prepared to make modeling with the ADeL more convenient and appealing. 
In contrast to ordinary (i.e., not extended) feature diagrams, which are validated based on propositional logic, the validation of ADeL deployment plans by the OCL introduces the expressiveness of predicate logic. Because of the promising results for extended feature diagrams [18], we intend to achieve an optimization of deployment plans by a transformation to constraint satisfaction problems.

\section{References}

[1] Altenhofen, M., Hettel, T., Kusterer, S.: OCL support in an industrial environment. In: Auletta, V. (ed.) MoDELS 2006. LNCS, vol. 4364, pp. 169-178. Springer, Heidelberg (2007)

[2] Aier, S., Riege, C., Winter, R.: Unternehmensarchitektur-Literaturüberblick und Stand der Praxis. WIRTSCHAFTSINFORMATIK 40, 292-304 (2008)

[3] Anderson, J.R.: Cognitive Psychology and its Implications, 5th edn. Worth, New York (2000)

[4] Budinsky, F., Steinberg, D., Merks, E., Ellersick, R., Grose, T.J.: Eclipse Modeling Framework: A Developer's Guide. Addison-Wesley, Boston (2004)

[5] Carzaniga, A., et al.: A Characterization Framework for Software Deployment Technologies. Techn. Report CU-CS-857-98, Dept. of Computer Science, University of Colorado (1998)

[6] Distributed Management Task Force (DMTF): Common Information Model (CIM) Standards. CIM Schema Version 2.24.0,

http: / /www.dmtf.org/standards/cim/cim_schema_v2240/(2010)

[7] Distributed Management Task Force (DMTF): CIM FAQ (2010),

http: / / dmtf.org/about/faq/cim_faq\#C4

[8] Frank, U., et al.: ITML: A domain-specific modeling language for supporting businessdriven IT Management. In: Proc. 9th OOPSLA Workshop on Domain-Specific Modeling. DSM Forum (2009),

http: / /www.dsmforum. org/events / DSM09/Papers / Heise.pdf

[9] Hall, R.S., Heimbigner, D., Wolf, A.L.: A Cooperative Approach to Support Software Deployment Using the Software Dock. In: Proc. 21st Int. Conf. on Software Engineering (ICSE 1999). ACM Press, New York (1999)

[10] Kruse, S., et al.: Decoupling Models and Visualisations for Practical EA Tooling. In: Dan, A., Gittler, F., Toumani, F. (eds.) ICSOC/ServiceWave 2009 Workshops. LNCS, vol. 6275, pp. 62-71. Springer, Heidelberg (2010)

[11] Lacour, S., Pérez, C., Priol, T.: Generic Application Description Model: Toward Automatic Deployment of Applications on Computational Grids. Rapport de Recherche No. 5733, INRIA, Rennes (2005)

[12] Lankhorst, M., et al.: Enterprise Architecture at Work: Modeling, Communication, Analysis. Springer, Berlin (2005)

[13] Lestideau, V., Belkhatir, N.: Providing highly automated and generic means for software deployment process. In: Oquendo, F. (ed.) EWSPT 2003. LNCS, vol. 2786, pp. 128-142. Springer, Heidelberg (2003)

[14] Makin, N.: Deployment modeling in Rational Software Architect Version 7.5, Part I \& II (2008), http: / / www. ibm.com/developerworks/rational /

library/08/\{1202|1230\}_makin/

[15] Object Management Group (OMG): Unified Modeling Language: Superstructure, Version 2.2, formal/2009-02-02 (2009), http : / / www . omg . org / 
[16] OMG: UML 2.0 OCL Specification, formal/2006-05-01 (2006),

http: / /www. omg.org /

[17] Patig, S., Herden, S., Zwanziger, A.: Modeling Deployment of Enterprise Applications Cases and Conclusions. Preprint No. 224, University of Bern, IWI (2009)

[18] Benavides, D., Trinidad, P., Ruis-Cortés, A.: Automated Reasoning on Feature Models. In: Pastor, Ó., Falcão e Cunha, J. (eds.) CAiSE 2005. LNCS, vol. 3520, pp. 491-503. Springer, Heidelberg (2005)

[19] Warner, J., Kleppe, A.: Object Constraint Language 2.0. Bonn: mitp (2004)

[20] Giese, S., Seibel, A., Vogel, T.: A Model-Driven Configuration Management System for Advanced IT Service Management. In: Proc. 4th Int. Workshop on Models@run.time at the 12th IEEE/ACM Int. Conf. on Model Driven Engineering Languages and Systems (MoDELS 2009). CEUR Workshop Proceedings, vol. 509, pp. 61-70 (2009)

[21] Galan, F., Lopez de Vergara, J., Fernandez, D., Munoz, R.: Scenario-based Configuration Management for Flexible Experimentation Infrastructures. In: Proc. 5th Int. IEEE Conf. on Testbeds and Research Infrastructures for the Development of Networks \& Communities. IEEE Press, Los Alamitos (2009),

http://doi.ieeecomputersociety.org/10.1109/ TRIDENTCOM. 2009.4976228

[22] Project Group: Topcased, Version 2.6.0, http: / / www . topcased.org

[23] Object Management Group (OMG): Unified Modeling Language: Infrastructure, Version 2.2, formal/2009-02-04 (2009), http: / /www . omg . org / 